This document is the accepted manuscript version of the following article:

Artus, G. R. J., Zimmermann, J., Reifler, F. A., Brewer, S. A., \& Seeger, S. (2012). A superoleophobic textile repellent towards impacting drops of alkanes. Applied Surface Science, 258(8), 3835-3840.

https://doi.org/10.1016/j.apsusc. 2011.12.041

\title{
A Superoleophobic Textile Repellent towards Impacting Drops of Alkanes
}

Georg R. J. Artus, ${ }^{\mathrm{a}}$ Jan Zimmermann, ${ }^{\mathrm{a} 1}$ Felix A. Reifler, ${ }^{\mathrm{b}}$ Stuart A. Brewer ${ }^{\mathrm{c}}$ and Stefan Seeger*a

${ }^{a}$ Institute of Physical Chemistry, University of Zurich, Winterthurerstrasse 190, 8057 Zurich, Switzerland. Fax: +41 44635 6813; Tel: +41 44635 4450; E-mail: g.artus@pci.uzh.ch, j.zimmermann@forsterrohner.com

* corresponding author sseeger@pci.uzh.ch

${ }^{\mathrm{b}}$ Laboratory for Advanced Fibers, Empa, Swiss Federal Laboratories for Materials Science and Technology, Lerchenfeldstrasse 5, 9014 St. Gallen, Switzerland. E-mail:

felix.reifler@empa.ch

${ }^{\mathrm{c}}$ Defence Science and Technology Laboratory, Dstl Porton Down, Salisbury, Wiltshire, SP4 0JQ, United Kingdom. E-mail: SABREWER@mail.dstl.gov.uk

\begin{abstract}
A commercially available polyester fabric has been rendered superoleophobic by coating with silicone nanofilaments and subsequent plasma fluorination. The treated samples show outstanding oil-repellency: They achieve the highest possible oil-repellency grade of 8 , repel impacting drops of alkanes and show a plastron layer in hexadecane. The oil repellency is shown to depend on the topography of the silicone nanofilament coating.
\end{abstract}

\section{Keywords}

Superoleophobic, Textile, Coating, Silicone, Nanofilaments, Shedding Angle

\footnotetext{
${ }^{1}$ Present address: Forster Rohner AG, Flurhofstrasse 150, Postfach 319, 9006 St. Gallen, Switzerland
} 


\section{Introduction}

The wetting of a solid surface by a liquid is a very important process in nature, science and technology. Of special scientific and economic interest are non-wetting materials. Especially textiles resistant to the spreading or penetration of liquids would find widespread applications. Water-repellent surfaces and also textiles have been developed during the last fifteen years [1-6]. With respect to e. g. fatty and oily substances in food manufacture or lubricants and fuels in engineering technology oleophobic materials are just as desirable as hydrophobic ones. The liquids of interest are common oils but also pure van-der-Waals liquids with surface tensions ranging from $35 \mathrm{mNm}^{-1}$ down to $20 \mathrm{mNm}^{-1}$. Liquids which such a low surface tension wet all common materials making the design of highly oil-repellent surfaces much more demanding compared to superhydrophobic surfaces [7]. Only recently the requirements for the design of highly oleophobic surfaces have emerged. First, an essential necessity is a multi-valued surface topography [7-9]. Second, the points of contact between solid and liquid should be as small as possible but densely arranged $[7,10]$. Third, the surface energy of the substrate should be as low as possible $[7,8,11]$. Due to these quite intricate prerequisites up to now only a few surfaces have been reported on which low surface tension liquids such as hexadecane $\left(\gamma=27 \mathrm{mNm}^{-1}\right)[12-16]$, dodecane $\left(\gamma=24.9 \mathrm{mNm}^{-1}\right)[16,17]$, decane $(\gamma=23.4$ $\left.\mathrm{mNm}^{-1}\right)$ [16], ethanol $\left(\gamma=22 \mathrm{mNm}^{-1}\right)$ [9], silicone oil $\left(\gamma=22 \mathrm{mNm}^{-1}\right)[14]$ or octane $(\gamma=21.1$ $\mathrm{mNm}^{-1}$ ) [8] show superoleophobicity, meaning apparent contact angles above $150^{\circ}$ and low sliding angles.

Some years ago, silicone nanofilaments (SNF) including a technique for applying them as coating have been developed in our group $[5,18]$. The coated surfaces exhibit pronounced superhydrophobic properties [6,19]. Due to the highly multi-valued topography of the SNF coating and the dense packing of nanometre-sized filaments, the coating matches all criteria for superoleophobicity after additional reduction of the surface energy by fluorination. This has been demonstrated by wet chemical fluorination of SNF coated glass e. g. with decane 
[16]. In this work we report a textile with outstanding superoleophobic properties. The samples achieve the highest possible oil-repellency grade (ORG) of 8, repel impacting drops of octane and are capable of stabilizing a plastron layer in alkanes. Comparison of samples with different densities of SNF reveals that the specific topography of the coating has a major effect on the oil repellency.

\section{Material and Methods}

The polyester fabric (\#407, polyester dacron, Type 54 spun, plain weave, $140 \mathrm{gm}^{-2}$, according to ISO 105-F04, EMPA Testmaterialien AG, Switzerland), methyltrichlorosilane (97\%), 1H, $1 \mathrm{H}, 2 \mathrm{H}, 2 \mathrm{H}$-perfluorooctyltrichlorosilane (PFOTS, both from ABCR, Germany), $n$ hexadecane, $n$-decane, $n$-octane and $n$-heptane (Fluka, Switzerland) and the monomer $\mathrm{C}_{8} \mathrm{~F}_{17} \mathrm{CH}_{2} \mathrm{CH}_{2} \mathrm{OCOCH}=\mathrm{CH}_{2}$ (PFAC8, Fluorochem, UK) were used as received. The detailed procedure for gas phase coating with silicone nanofilaments is described in the literature [5,6]. In brief: The polyester fabric was cut into pieces of approx. $10 \mathrm{~cm}$ by $20 \mathrm{~cm}$ in size. The coatings were performed in the gas phase in a desiccator by exposure of the textiles to vapour of methyltrichlorosilane $(595 \mathrm{mg} \pm 1 \mathrm{mg}$ ) at normal pressure and room temperature. Increasing filament densities could be obtained by adjusting different relative humidity values in the coating chamber ranging from $26 \%$ to $40 \%$.

For plasma fluorination PFAC8 was used as monomer at a pressure of 0.1 torr and $30^{\circ}$ temperature in the reactor [20,21]. A plasma peak power of $40 \mathrm{~W}$, a plasma on/off cycle of 20 $\mu \mathrm{s}$ on and an off time of $20 \mathrm{~ms}$ were used. The optimum plasma treatment time was found to be $90 \mathrm{~s}$.

For the determination of shedding angles pieces of approx. $2 \mathrm{~cm}$ by $5 \mathrm{~cm}$ were cut out and carefully attached to glass slides using double sided adhesive tape. Measurements were performed with the Contact Angle System OCA (Dataphysics, Germany) and a custom-made tilting device according to the literature [22]. Experiments were started at an inclination angle 
of $70^{\circ}$. When at least five drops rolled off completely after impact the inclination angle was decreased by $5^{\circ}$ and the measurement repeated. The drop volume was checked by weighing 10 drops. The drop volumes were $6.5 \mu \mathrm{L} \pm 0.2 \mu \mathrm{L}$ for hexadecane, $6.2 \mu \mathrm{L} \pm 0.3 \mu \mathrm{L}$ for dodecane, $6.1 \mu \mathrm{L} \pm 0.2 \mu \mathrm{L}$ for decane and $6.1 \mu \mathrm{L} \pm 0.2 \mu \mathrm{L}$ for octane, respectively. The reported shedding angles are averaged per sample type.

The evaluation of the ORGs was performed in the style of ISO 14419:1998 [23]. Prior to testing, the test specimens were conditioned for a minimum of $4 \mathrm{~h}$ at a temperature of $20^{\circ} \mathrm{C} \pm$ $2{ }^{\circ} \mathrm{C}$ and a relative humidity of $65 \% \pm 2 \%$. For evaluation regarding a specific ORG, a drop of the respective test liquid was carefully placed on the surface of the test specimen. Its shape and wetting behaviour were assessed after $30 \mathrm{~s} \pm 2 \mathrm{~s}$ and further observed for some more minutes (see also figures $\mathrm{S} 1$ and $\mathrm{S} 2$ in the electronic supplementary material).

For electron microscopy small samples of approx. $4 \mathrm{~mm}$ by $4 \mathrm{~mm}$ in size were cut out and attached on metal sample holders with conducting adhesive tabs. A trace of carbon glue was added to reduce charging. Rotating samples were sputter coated with $15 \mathrm{~nm}$ of platinum. Electron microscopy images were taken at the Zeiss Supra $50 \mathrm{VP}$ at the centre for microscopy and image analysis of the University of Zurich at $2 \mathrm{kV}$ acceleration voltage.

\section{Results and Discussion}

Samples were prepared by coating pieces of a polyester textile with SNF and subsequent plasma fluorination according to the literature [5,6,20,21]. Higher densities of the SNF coating were achieved by adjusting a higher humidity in the coating chamber.

For the determination of the oleophobicity the ORGs according to ISO 14419, a common rating method in textile industry, were determined [23]. Drops of eight different test liquids with decreasing surface tensions are placed onto the sample. An ORG on the scale between zero and eight is assigned by determining the corresponding test liquid that does not show any penetration, wetting or wicking after 30 seconds. The test liquid for grade six is decane, for 
grade seven it is octane and for grade eight it is heptane. The obtained ORGs vary between 6 and 8 depending on the quality of the SNF coating. We therefore classified all samples into three categories depending on the ORG. Figure 1 displays scanning electron microscopy images of polyester fibres of the uncoated reference sample (a) and samples with ORG 6, 7 and 8 ( $b$ to d). It is obvious that the degree of coverage, coating thickness, length and density of the SNF all increase from samples with ORG 6 to 8.

Interestingly, the reference sample without the SNF coating also achieves a very good ORG of 7. Obviously, the incomplete coverage and low density of the SNF on the polyester fibres of the samples with ORG 6 aids the penetration of the liquid front into the sample for octane and heptane instead of preventing it. This is well described by the homogeneous Wenzel-type of wetting which predicts improved wetting due to moderate roughness on a hygrophilic material [24]. For samples with an ORG of 7 the SNF have no detectable effect on the oil repellency compared to the reference. From the improvement of the ORG for samples with an ORG of 8 it is obvious that a dense SNF coating enhances the oleophobicity. The significance of this effect becomes apparent during the ORG measurements: Contrary to the reference sample, drops of octane move with ease across samples with ORG 8 and did not get pinned. These results are well reflected by the measured contact and sliding angles with hexadecane on samples with an ORG of 8 . Contact angles above $150^{\circ}$ and sliding angles below $15^{\circ}$ can be obtained routinely.

This extremely repellent behaviour is due to a heterogeneous wetting state of the liquid [25]. The drop does not penetrate the surface structure but instead resides only on the highest asperities of the rough surface. This reduces the effective area of the liquid-solid interface while most of the apparent supporting area consists of an interface liquid-air. The contact angles of this heterogeneous wetting state are well described by the Cassie-Baxter equation [26]. It is well known that a multiscale roughness benefits high contact angles [25,27,28]. A multiscale roughness certainly is present on our samples: The textile itself provides a 
macroscale roughness given by the diameter of the yarn of approx. $200 \mu \mathrm{m}$. The yarn itself consists of single microscopic polyester fibres with a diameter of $12 \mu \mathrm{m}$ and finally, these polyester fibres are decorated with SNF providing roughness on the sub-micron scale. However, this multiscale roughness does not contribute much to the high contact angles observed on our samples. This can be concluded from contact angle data which have been measured on flat glass substrates coated with fluorinated SNF [16]. Contact angles well above $150^{\circ}$ and sliding angles below $5^{\circ}$ are reported for several solvents with low surface tension, also hexadecane. These high contact angles indicate that the SNF-coating alone suffices for obtaining high contact angles and that additional micro- or macroscale roughness plays a minor role. For the investigation of the effects of roughness on the different length-scales a comparison of measured contact angles and theoretically calculated contact angles via the Cassie-Baxter equation could be instructive. Unfortunately this comparison fails due to the impossibility of determining contact angles on macroscopically rough and non-reflecting samples as on our textile. This problem is well documented in the literature [22,29] and also conforms to our experience (see figure S3 in the electronic supplementary material). For this reason we want to forgo any further discussion of contact angles.

Furthermore, the contact angle is only a measure of the static wettability. At least as important is the robustness of the heterogenous non-wetting state with respect to penetration of the liquid into the substrate $[10,17]$. Fading into the homogenous wetted state would imply complete loss of the repellent properties [30]. For liquids with low surface tension this transition is even more problematic, since contrary to water the thermodynamically most stable state for these liquids is the completely wetted state in any case [7]. Penetration of the drop can be triggered by oleophilic defects at the solid surface, by capillary waves or by enhanced pressure in the liquid [25,27]. Elevated pressure may be caused by Laplace pressure inside very tiny drops, hygrostatic pressure because of a bulk liquid phase on top of a surface, or during impact of a drop. In order to gain information about the robustness and the 
penetration behaviour of an impacting drop we measured the shedding angle $\omega$ with several alkanes. A detailed description and discussion of this experimental method is given in the literature [22]. In brief, the sample is mounted on a table with an adjustable inclination angle. A drop of a test liquid with known volume is released at a defined height above the sample. The shedding angle is the lowest inclination angle of the substrate, where the droplet rolls off completely at least five times at different points of impact. Clearly the shedding angle is a measurement on a dynamic system, whereas the contact angle and the ORG provide data on a static system. So the shedding angle is a measure for two very important properties of the surface, the dynamic wetting behaviour and the robustness of the surface towards penetration of the liquid.

Figure 2 displays the shedding angles for low surface tension alkanes measured on SNF coated and plasma-fluorinated polyester fabric samples. A shedding angle below or equal to $70^{\circ}$ cannot be observed on neither the reference sample nor samples with an ORG of 6 with either test liquid. Although the reference is highly repellent in static wetting situations as indicated by an ORG of 7, the dynamic oleophobicity is bad. Further reduction of the surface energy on the reference sample by prolonged periods of plasma fluorination did not show any improvements. From this, we conclude that a low surface energy is not sufficient to improve the dynamic repellency properties. Rather the specific surface topography of the SNF coating seems to be more important. This becomes already obvious for samples with an ORG of 7: Although these samples and the reference display the same static repellency as indicated by the common ORG of 7, shedding angles can only be measured for the coated samples, albeit not for octane. The effect of the surface topography is even more pronounced for the samples with an ORG of 8, which show exceptional dynamic oil repellent properties. As can be expected the shedding angle behaves inversely to the surface tension of the test liquid. With hexadecane and dodecane very good shedding angles below $25^{\circ}$ are achieved. For decane the shedding angle is $30^{\circ}$. Even drops of octane with a low surface tension of $21.1 \mathrm{mNm}^{-1}$ do not 
adhere to the sample but roll off the surface after impact at an inclination angle of $48^{\circ}$ (see also movie M1 in the electronic supplementary material). The surface is capable of withstanding a drop of octane not only when it is placed gently onto the surface but also upon drop impact. This is a very important result since repellency in dynamic wetting situations, such as impacting drops, will be a prerequisite for application of superoleophobic textiles. Just static repellency will not suffice. To our knowledge such dynamic oil repellent properties on a textile are unprecedented.

On these samples with an ORG of 8 a drop of heptane will roll off after impact at higher inclination angles. But too often there remains a small trail of liquid on the fabric. Therefore no shedding angle is reported. Nevertheless, one certainly would assign a dynamic repellent behaviour towards this liquid.

Besides impact of a drop also subjection to hygrostatic pressure can reveal the robustness of a repellent surface against penetration. Upon immersion of a superhydrophobic sample in water a plastron is formed. The air layer between solid and water is visible as a shiny silvery layer due to total internal reflection of light. This effect is well known for natural and artificial surfaces $[19,31,32]$. The existence of a plastron layer between our superoleophobic textile samples and alkanes is demonstrated in figure 3. The left image shows an uncoated textile immersed in hexadecane. The fabric is completely wetted by the liquid. The middle image of figure 3 displays a sample with an ORG of 8 immersed in hexadecane, and in the right image immersed in heptane. The plastron layer is clearly visible for both solvents. In hexadecane the gas layer is evenly distributed all over the sample. It is very similar to the plastron layer observed for the superhydrophobic version of the same polyester fabric immersed in water [6]. The plastron is stable for minutes as indicated by continuous total reflection of light. After removal from the solvent the fabric is not soaked or wetted by the liquid although some drops remained adhered to the fabric. The existence and stability of a plastron layer in the low surface tension organic solvent hexadecane is remarkable. To our knowledge this is the first 
demonstration of a stable large area plastron layer between a superoleophobic textile surface and an organic solvent with a low surface tension $[15,16]$. According to the shedding angles this result underlines the outstanding superoleophobic properties of this kind of sample. In heptane the plastron is also clearly visible. In this case however, the gas layer appears to be less homogeneous and partially disappeared within a few minutes. After removal, the fabric was partially wetted.

The robustness of the presented textile towards penetration of low-surface-tension liquids is due to several reasons. As presented above the surface is rough on several length scales. Furthermore, the hierarchical arrangement of yarn, polyester fibres and SNF results in a highly multi-valued surface topography as it is needed for oil-repellency [8,9]. A more mathematical inspection of the benefits of a multi-valued topography revealed that the existence of surfaces with a convex curvature is the decisive point for stabilising the heterogeneous wetting state [7]. All parts of the surface of our samples are convexly curved due to the cylindrical shape of polyester fibres and SNF. Moreover, these structural components are mostly unsupported and can be approximated as free standing cylinders. Concave sections only occur where two cylinders are in direct contact. Here we see an important difference of our sample compared to many other superoleophobic surfaces. Most surface designs achieve multi-valued topography by piling or stacking convexly shaped structural elements on top of each other. For example this is the case for nano-nails [9] or textiles rendered superoleophobic by attaching silica particles of different diameters [13]. These arrangements necessarily imply much more concave sections of the surface. The consequences of this are depicted in figure 4 . The liquid front of a drop sitting on top of a superoleophobic surface is stable when the surface is convexly shaped. This is depicted for a pressure $p_{1}$ for supported and unsupported convex structural components, e. g. cylinders or spheres. The three-phase-contact line is positioned at point A where the contact angle $\theta_{\mathrm{Y}}$ is formed [7]. The liquid volume extends to the dark grey area limited by the meniscus given as 
continuous line. The radii of curvature of all menisci are much larger than the size of the nanoscale structural components of the surface. Therefore we can approximate the meniscus as a straight line. Enhancing the pressure to $p_{2}$ makes the liquid-air interface bulge downwards (dashed line). The contact line advances further along the solid since the local contact angle $\theta_{\mathrm{Y}}$ has to remain constant. For the supported structure this advancement of the contact line facilitates further penetration. At pressure $p_{2}$ the contact line may have reached point $\mathrm{B}$ where the surface curvature changes its sign to become concave. At concave curvature of the solid the contact line is no longer stable and thus may easily advance further [7]. Also the corresponding meniscus at pressure $p_{2}$ may come close enough to the supporting structure at point $\mathrm{C}$ to get into contact. As a consequence penetration would proceed. The next stable wetting situation at the released pressure $p_{1}$ is reached when the contact line has advanced to point D (dotted line) and the liquid volume has filled the area marked in light grey. Contrary, for the unsupported structure the advancement of the contact line to point B has no consequences. Penetration can only proceed when the two liquid volumes advancing on both sides of the structure come close enough to unite at the lower apex at point E (light grey area, dash-dotted menisci). This would require a much higher pressure $p_{3}$. In other words, the energy barrier which has to be overcome for advancement of the liquid front is higher for unsupported structural elements than for a supported arrangement. High energy barriers for the transition from the heterogeneous to the homogeneous wetting are indispensible for a high robustness of superoleophobic surfaces [8].

Besides the height of the energy barriers a high number or density of energy barriers will contribute to the robustness. Also this is satisfied in our sample by the high number of SNF. Increasing density and length of the SNF imply a multitude of potential pinning points each separated by an energy barrier. This effect is reflected by the dependency of ORG and shedding angle on the density of the SNF-coating. 


\section{Conclusion}

We have rendered a textile superoleophobic by coating it with silicone nanofilaments and subsequent plasma fluorination. The coated fabric achieves the highest possible oil repellency grade of eight. As revealed by measurement of the shedding angles the fabric not only repels static drops of liquids of low surface tension, but is also capable of repelling impacting drops. A stable plastron is formed at the interface between the textile and hexadecane. The oilrepellent properties depend on the density and length of the silicone nanofilaments. Among the desired applications of superhydrophobicity are reduced adhesion of dirt particles and self-cleaning surfaces, reduction of viscous drag, waterproof textiles and waterproof surfaces for anti-fouling or anti-corrosion purposes. With the latest advances in superoleophobicity research all these applications can now be extended from water to oily liquids. Of primary interest are applications in engineering wherever lubricants or fuels are used. In addition, self-cleaning surfaces relevant for organic solvents and a reduction of viscous drag for microfluidic devices that use non-aqueous solvents, could allow completely new applications in technology to be considered. Also the exploration of plastrons in nonaqueous environments could be worthwhile. We believe that the silicone nanofilament coating may contribute to these developments due to its suitability for the creation of superoleophobic surfaces and the ease with which it can be applied to a wide variety of materials.

\section{Acknowledgements}

We thank the centre for microscopy and image analysis of the University Zurich for their support and for the possibility to use their facilities.

\section{References}

[1] P. Roach, N. J. Shirtcliffe, M. I. Newton, Progress in superhydrophobic surface development, Soft Matter 4 (2008) 224-240. 
[2] X.-M. Li, D. Reinhoudt, M. Crego-Calama, What do we need for a superhydrophobic surface? A review on the recent progress in the preparation of superhydrophobic surfaces, Chem. Soc. Rev. 36 (2007) 1350-1368.

[3] I. P. Parkin, R. G. Palgrave, Self-cleaning coatings, J. Mater. Chem. 15 (2005) 1689-1695.

[4] X. J. Feng, L. Jiang, Design and Creation of Superwetting/Antiwetting Surfaces, Adv. Mater. 18 (2006) 3063-3078.

[5] G. R. J. Artus, S. Jung, J. Zimmermann, H. P. Gautschi, K. Marquardt, S. Seeger, Silicone Nanofilaments and Their Application as Superhydrophobic Coatings, Adv. Mater. 18 (2006) $2758-2762$.

[6] J. Zimmermann, F. A. Reifler, G. Fortunato, L.-C. Gerhardt, S. Seeger, A Simple, OneStep Approach to Durable and Robust Superhydrophobic Textiles, Adv. Funct. Mater. 18 (2008) 3662-3669.

[7] A. Marmur, From Hygrophilic to Superhygrophobic: Theoretical Conditions for Making High-Contact-Angle Surfaces from Low-Contact-Angle Materials, Langmuir 24 (2008) 75737579.

[8] A. Tuteja, W. Choi, M. Ma, J. M. Mabry, S. A. Mazzella, G. C. Rutledge, G. H. McKinley, R. E. Cohen, Designing Superoleophobic Surfaces, Science 318 (2007) 16181622.

[9] A. Ahuja, J. A. Taylor, V. Lifton, A. A. Sidorenko, T. R. Salamon, E. J. Lobaton, P. Kolodner, T. N. Krupenkin, Nanonails: A Simple Geometrical Approach to Electrically Tunable Superlyophobic Surfaces, Langmuir 24 (2008) 9-14.

[10] S. S. Chhatre, W. Choi, A. Tuteja, K.-C. Park, J. M. Mabry, G. H. McKinley, R. E. Cohen, Scale Dependence of Omniphobic Mesh Surfaces, Langmuir 26 (2010) 4027-4035.

[11] S. S. Chhatre, A. Tuteja, W. Choi, A. Revaux, D. Smith, J. M. Mabry, G. H. McKinley, R. E. Cohen, Thermal Annealing Treatment to Achieve Switchable and Reversible Oleophobicity on Fabrics, Langmuir 25 (2009) 13625-13632. 
[12] A. Steele, I. Bayer, E. Loth, Inherently Superoleophobic Nanocomposite Coatings by Spray Atomization, Nano Lett. 9 (2009) 501-505.

[13] B. Leng, Z. Shao, G. de With, W. Ming, Superoleophobic Cotton Textiles, Langmuir 25 (2009) 2456-2460.

[14] W. Wu, X. Wang, D. Wang, M. Chen, F. Zhou, W. Liu, Q. Xue, Alumina nanowire forests via unconventional anodization and super-repellency plus low adhesion to diverse liquids, Chem. Commun. (2009) 1043-1045.

[15] H. Jin, M. Kettunen, A. Laiho, H. Pynnönen, J. Paltakari, A. Marmur, O. Ikkala, R. H. A. Ras, Superhydrophobic and Superoleophobic Nanocellulose Aerogel Membranes as Bioinspired Cargo Carriers on Water and Oil, Langmuir 27 (2011) 1930-1934.

[16] J. Zhang, S. Seeger, Superoleophobic Coatings with Ultralow Sliding Angles Based on Silicone Nanofilaments, Angew. Chem. Int. Ed. 50 (2011) 6652-6656.

[17] W. Choi, A. Tuteja, S. Chhatre, J. M. Mabry, R. E. Cohen, G. H. McKinley, Fabrics with Tunable Oleophobicity, Adv. Mater. 21 (2009) 2190-2195.

[18] J. Zimmermann, S. Seeger, G. R. J. Artus, S. Jung, Superhydrophobic Coating, World Pat. 2004/113456.

[19] J. Zimmermann, G. R. J. Artus, S. Seeger, Superhydrophobic Silicone Nanofilament Coatings, J. Adhes. Sci. Technol. 22 (2008) 251-263.

[20] S. R. Coulson, I. S. Woodward, J. P. S. Badyal, S. A. Brewer, C. Willis, Plasmachemical Functionalization of Solid Surfaces with Low Surface Energy Perfluorocarbon Chains, Langmuir 16 (2000) 6287-6293.

[21] S. R. Coulson, I. S. Woodward, J. P. S. Badyal, S. A. Brewer, C. Willis, Ultralow Surface Energy Plasma Polymer Films, Chem. Mater. 12 (2000) 2031-2038.

[22] J. Zimmermann, S. Seeger, F. A. Reifler, Water Shedding Angle: A new technique to evaluate the water repellent properties of superhydrophobic surfaces, Text. Res. J. 79 (2009) $1565-1570$. 
[23] International Organization for Standardization, Textiles - Oil repellency - Hydrocarbon resistance test, ISO 14419, Geneva, 1998.

[24] R. N. Wenzel, Resistance of Solid Surfaces to Wetting by Water, Ind. Eng. Chem. 28 (1936) 988-994.

[25] B. Bhushan, Y. C. Jung, Natural and biomimetic artificial surfaces for superhydrophobicity, self-cleaning, low adhesion, and drag reduction, Prog. Mater. Sci. 56 (2011) 1-108.

[26] A. B. D. Cassie, S. Baxter, Wettability of Porous Surfaces, Trans. Faraday Soc. 40 (1944) 546-551.

[27] M. Nosonovsky, B. Bhushan, Hierarchical roughness makes superhydrophobic states stable, Microelectron. Eng. 84 (2007) 382-386.

[28] Y. Su, B. Ji, K. Zhang, H. Gao, Y. Huang, K. Hwang, Nano to Micro Structural Hierarchy Is Crucial for Stable Superhydrophobic and Water-Repellent Surfaces, Langmuir 26 (2010) 4984-4989.

[29] A. Marmur, E. Bittoun, When Wenzel and Cassie Are Right: Reconciling Local and Global Considerations, Langmuir 25 (2009) 1277-1281.

[30] J. B. Boreyko, C. H. Baker, C. R. Poley, C.-H. Chen, Wetting and Dewetting Transitions on Hierarchical Superhydrophobic Surfaces, Langmuir 27 (2011) 7502-7509.

[31] K. Koch, H. F. Bohn, W. Barthlott, Hierarchically Sculptured Plant Surfaces and Superhydrophobicity, Langmuir 25 (2009) 14116-14120.

[32] N. J. Shirtcliffe, G. McHale, M. I. Newton, C. C. Perry, F. B. Pyatt, Plastron properties of a superhydrophobic surface, Appl. Phys. Lett. 89 (2006) 104106. 


\section{Figures}

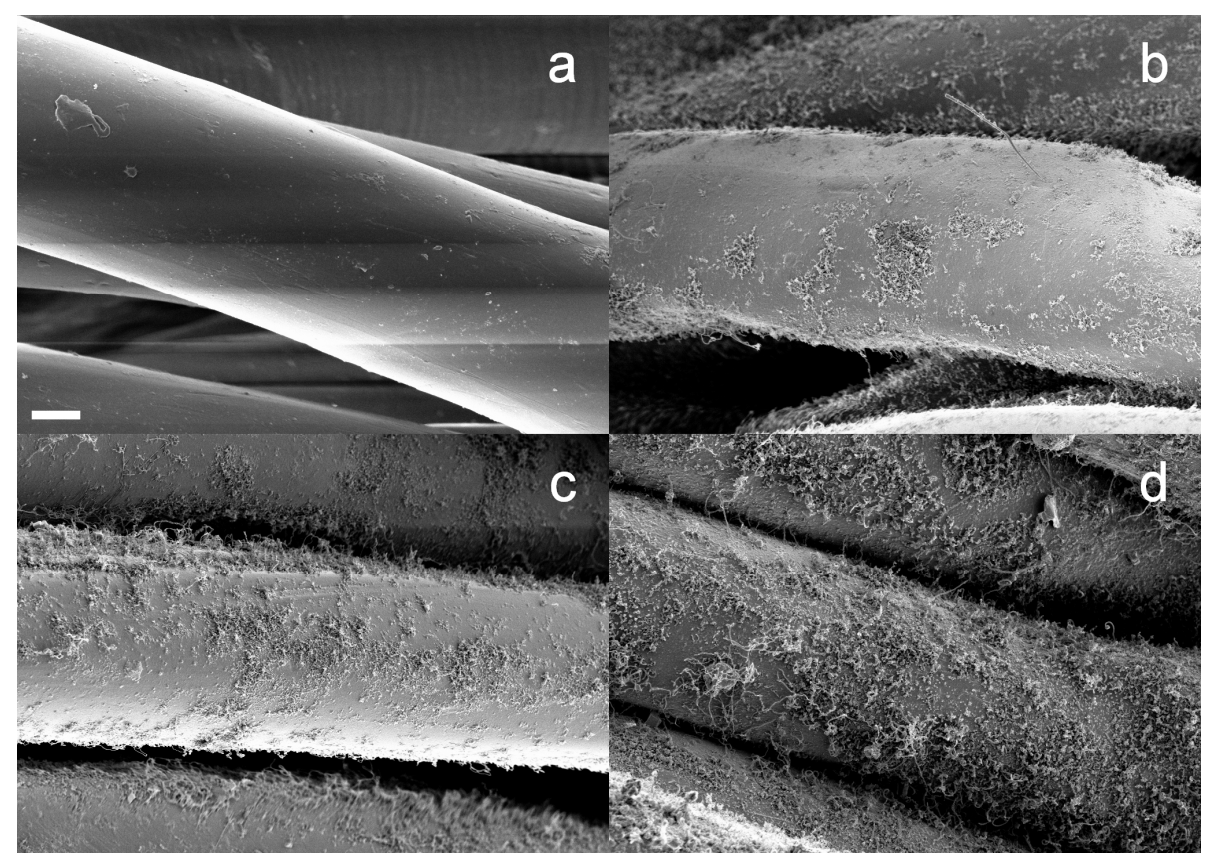

Fig. 1. Scanning electron microscopy images of polyester fabric samples: a) reference sample without SNF coating, b) sample with an ORG of 6 with a low density SNF coating, c) sample achieving an ORG of 7 with a medium density SNF coating, d) sample with the highest possible ORG of 8 with a high density SNF coating. The scale bar in figure a corresponds to 3 $\mu \mathrm{m}$ (same magnification for $\mathrm{b}$ to $\mathrm{d}$ ). 


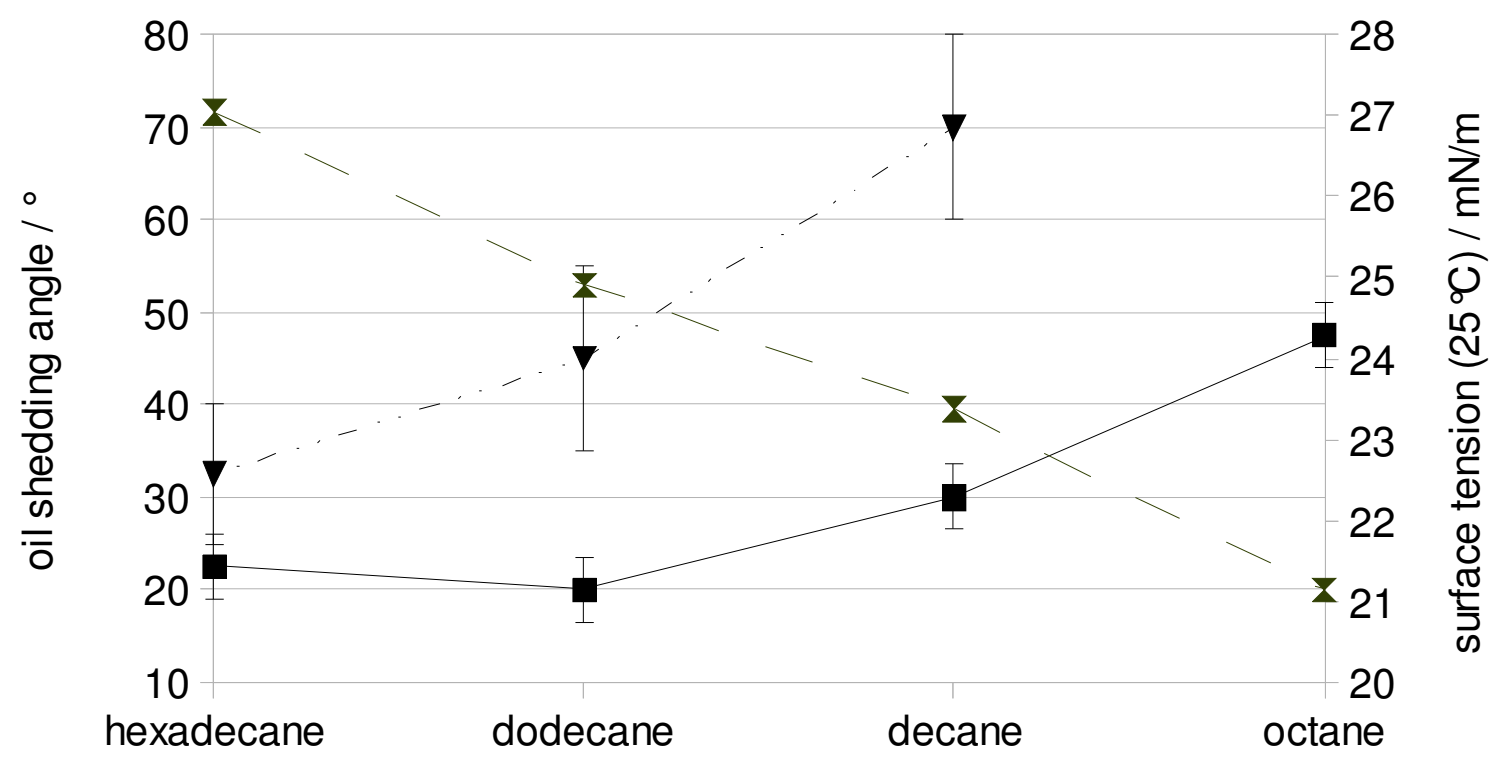

Fig. 2. Shedding angles for different $n$-alkanes on textile samples. Squares correspond to samples with ORG 8, triangles correspond to samples with ORG 7 (all left ordinate). The alkanes are arranged according to decreasing surface tension (double triangles, right ordinate). The connecting lines are just a guide to the eye. 


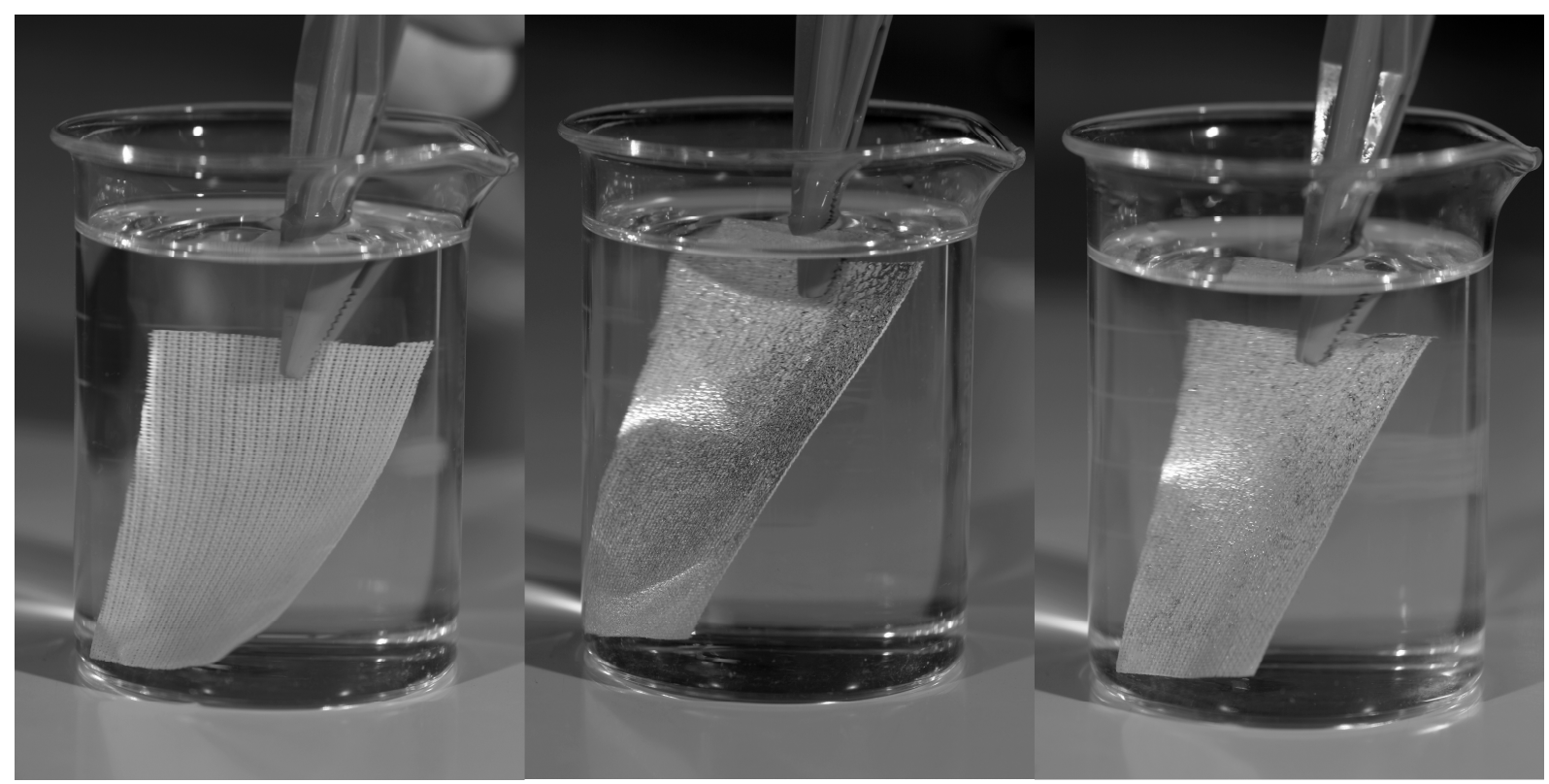

Fig. 3. Untreated piece of polyester fabric immersed in hexadecane as reference (left), sample with ORG 8 immersed in hexadecane (middle) and heptane (right) exhibiting a plastron layer in both solvents. 

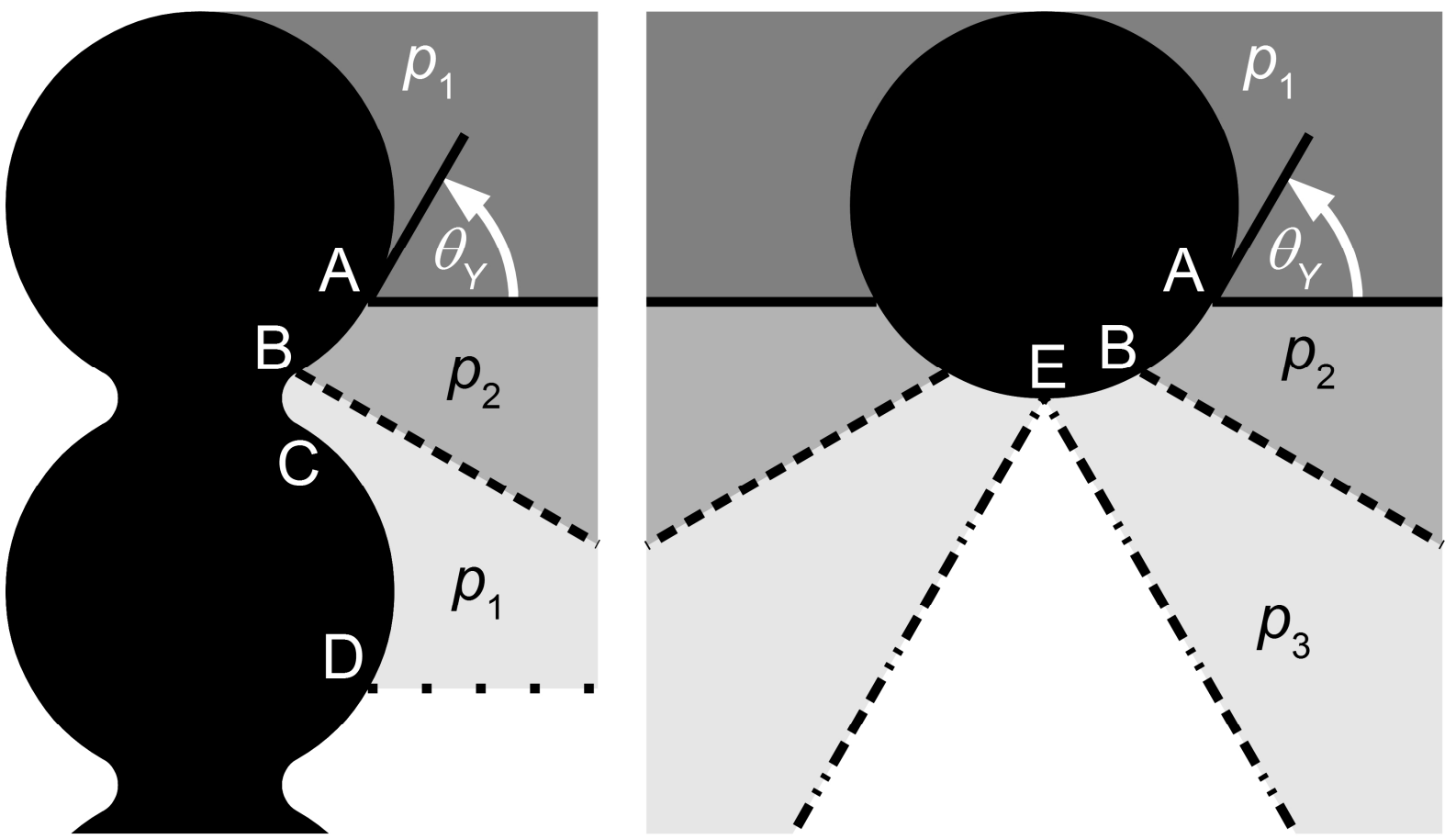

Fig. 4. Schematic representation of pressure induced advancement of a liquid front at supported (left) and unsupported (right) structural components (for explanations see text). 